\title{
New Bioanalytical Technologies in Platelet Research and Diagnostics
}

\author{
Peter Bugert \\ Institute of Transfusion Medicine and Immunology, Faculty of Clinical Medicine, University of Heidelberg, Mannheim, Germany
}

During the past years new technologies have been developed for the analysis of biomolecules such as nucleic acids or proteins. These technologies are characterized by increasing complexity or parallelism, miniaturization and automation. Primary goal of the new bioanalytic approaches is a large-scale molecular characterization and differentiation of certain types of molecules such as genomic DNA, mRNA or cytoplasmic proteins. Especially in the so-called postgenomic era after the sequencing of genomes from several species, including man, has been completed, further developments of bioanalytical technologies focused on a broad characterization of gene transcription and protein expression. Modern terms, such as 'transcriptomics' or 'proteomics', refer to the investigated class of molecules and the significant complexity of the data. Based on these technologies the biological sciences are undergoing a certain revolutionary change from intense studies of particular molecules to a rather broad investigation of molecular patterns to gain knowledge about cellular pathways and mechanisms.

DNA microarrays ('gene chips') represent the most important technology in the field of transcriptomics. This approach is now a robust, high-throughput, cost-effective technology capable of simultaneously quantifying tens of thousands of defined mRNA species in a miniaturized, automated format. Recently, the profiling of gene transcripts has been performed also in peripheral blood cells such as lymphocytes [1], neutrophils [2] and platelets [3-5]. The purification of platelet RNA posed a particular challenge with regard to obtaining sufficient amounts for microarray analysis and to contamination by leukocyte RNA. Both problems could be solved so that platelet RNA profiling can also be performed on clinical samples with limited platelet numbers $[6,7]$. The review article by Gnatenko and Bahou [8] in the present issue of TRANSFUSION MEDICINE AND HEMOTHERAPY summarizes current technical approaches to analyze transcriptomes. The authors also give an overview on functional categories of gene tran- scripts identified in normal platelets. In addition, recent findings concerning novel pathomechanisms of platelet disorders (e.g. grey platelet syndrome or essential thrombocytemia) identified by the use of transcriptomics are highlighted.

Compared to transcriptomics, measuring the complete protein profile (proteome) of a cell requires a much more labor intensive methodology which includes several steps of protein separation, fragmentation and mass spectrometrical analysis. The review article by Moebius et al. [9] gives an overview of the most important methods (gel-based or HPLC-based approaches), including a critical discussion of benefits and limitations of the different methods. Because many of them have been applied also to the platelet proteome, the review article summarizes the most important studies and their corresponding technological background. It is interesting that studies of different platelet subproteomes (phosphoproteome, membrane proteome, etc.) have been published recently and are considered in this review as well. In conclusion, the most important impact of proteomics in platelet research is the identification of a significant number of novel proteins (with known or unknown function) which have not been described for platelets before. The integration of both transcriptome and proteome data is reasonable, and both may contribute to the identification of novel proteins. Another review article in this issue highlights the limitations of transcriptome and proteome data, but both together can complement one another in order to obtain an almost complete view on proteins in platelets [10].

Especially in the platelet field, the complex analysis of gene transcripts and proteins is almost exclusively relevant for research purposes but not for the routine application in diagnostic laboratories. A current challenge of new bioanalytical technologies in the field of genomics is the complex typing of DNA polymorphisms, predominantly 'single nucleotide polymorphisms' (SNPs). Such technologies may have a significant

\begin{tabular}{|c|c|}
\hline KARGER & (C) 2006 S. Karger GmbH, Freiburg \\
\hline $\begin{array}{l}\text { Fax +49 } 7614520714 \\
\text { E-mail Information@Karger.de } \\
\text { www.karger.com }\end{array}$ & $\begin{array}{l}\text { Accessible online at: } \\
\text { www.karger.com/tmh }\end{array}$ \\
\hline
\end{tabular}

Peter Bugert, Ph.D.

Institut für Transfusionsmedizin und Immunologie

DRK-Blutspendedienst Baden-Württemberg - Hessen gGmbH

Friedrich-Ebert-Straße 107, 68167 Mannheim, Germany

Tel. +49 621 3706-8122, Fax -851

E-mail p.bugert@iti-ma.blutspende.de 
impact on platelet diagnostics in the future with regard to genotyping human platelet alloantigens (HPAs). In the review article by de Haas et al. [11] current 'medium throughput' methods for genotyping of HPA and blood groups are summarized with an emphasis on typing strategies in the Netherlands. The authors also provide an overview on the international developments of complex high-throughput SNP typing. This overview refers to four papers published in 2005 on the use of microarrays or beads carrying allele-specific oligonucleotide probes for genotyping of HPA and blood groups. Another high-throughput method was based on the use of tagged primers and single base extension. The aim of all studies on high-throughput typing strategies is to implement them into regular blood donor typing in order to achieve a large panel of fully typed blood donors. Further matching of blood transfusion as much as possible will prevent the occurrence of alloimmunization of blood recipients.

Significant progress has also been achieved in the diagnostic field of platelet immunology. The recent introduction of the simultaneous analysis of specific platelet antibodies (SASPA) assay [12] and of recombinant platelet antigens [13] represent the most important developments. Such methods may contribute to gain knowledge about the heterogeneity of platelet- specific antigens and antibodies which represents a significant problem in the clinical diagnosis of immunologically mediated platelet disorders. The current knowledge in this special field is addressed by the review article of Socher et al. [14] in which also a new technical approach, the surface plasmon resonance (SPR) technology, is introduced [14]. SPR allows the real-time measurement of antigen-antibody binding characteristics [15] and may help to improve platelet alloantibody diagnosis. In their review article Nguyen and Klüter [16] describe the benefits of the SASPA assay which represents a flow cytometrical method capable of the simultaneous detection and differentiation of several antibody specificities (platelet glycoproteins or HLA class I) and Ig subclasses (IgG or IgM). The implementation of this novel technique into routine diagnostic laboratory use and the 6-month experience of routinely performing the assay is reported as well.

New bioanalytical technologies for analysis of cellular genomes, transcriptomes and proteomes already show a significant impact on platelet research. In the field of clinical platelet diagnosis, high-throughput genomic approaches (including HPA and blood group typing) and complex antigenantibody analysis (e.g. SASPA assay) will be increasingly important in the future.

\section{References}

1 Stenz FB, Kitabchi AE: Transcriptome and proteome expression in activated human CD4 and CD8 T-lymphocytes. Biochem Biophys Res Commun 2004;324:692-696.

2 Roy S, Khanna S, Yeh PE, Rink C, Malarkey WB, Kiecolt-Glaser J, Laskowski B, Glaser R, Sen CK: Wound site neutrophil transcriptome in response to physiological stress in young men. Gene Expr 2005; 12:273-287.

3 Gnatenko DV, Dunn JJ, McCorkle SR, Weissmann D, Perrotta PL, Bahou WF: Transcript profiling of human platelets using microarray and serial analysis of gene expression. Blood 2003;101:2285-2293.

4 Bugert P, Dugrillon A, Günaydin A, Eichler H, Klüter H: Messenger RNA profiling of human platelets by microarray hybridization. Thromb Haemost 2003;90:738-748.

5 McRedmond JP, Park SD, Reilly DF, Coppinger JA, Maguire PB, Shields DC, Fitzgerald DJ: Integration of proteomics and genomics in platelets: a profile of platelet proteins and platelet-specific genes. Mol Cell Proteomics 2004;3:133-144.
6 Rox JM, Bugert P, Müller J, Schorr A, Hanfland P, Madlener P, Klüter H, Pötzsch B: Gene expression analysis in single donor platelets: evaluation of a PCR-based amplification technique. Clin Chem 2004;50:2271-2278.

7 Rolf N, Knoefler R, Suttorp M, Klüter H, Bugert P: Optimized procedure for platelet RNA profiling from blood samples with limited platelet numbers. Clin Chem 2005;51:1078-1080.

8 Gnatenko DV, Bahou WF: Recent advances in platelet transcriptomics. Transfus Med Hemother 2006;33:217-226.

9 Moebius J, Zahedi RP, Sickmann A: Platelet proteomics: essentials for understanding and application. Transfus Med Hemother 2006;33:227-235.

10 Bugert P, Ficht M, Klüter H: Towards the identification of novel platelet receptors: comparing RNA and proteome approaches. Transfus Med Hemother 2006:33.236-243.

11 de Haas M, van der Schoot CE, Beiboer SHW, Feskens M, Cheroutre G, Maaskant-van Wijk PA: Red blood cell and platelet genotyping: from current practice to future high-throughput donor typing. Transfus Med Hemother 2006;33:260-266.
12 Nguyen XD, Dugrillon A, Beck C, Kerowgan M, Klüter $\mathrm{H}$ : A novel method for simultaneous analysis of specific platelet antibodies: SASPA. Br J Haematol 2004;127:552-560.

13 Kroll H, Yates, Santoso S: Immunization against a low-frequency human platelet alloantigen in fetal alloimmune thrombocytopenia is not a single event: characterization by the combined use of reference DNA and novel allele-specific cell lines expressing recombinant antigens. Transfusion 2005; 45:353-358.

14 Socher I, Kroll H, Santoso S: Heterogeneity of platelet alloantigens and alloantibodies: new insights into structure and function. Transfus Med Hemother 2006;33:244-253.

15 Kimura S, Yurugi K, Segawa H, Kuroda J, Sato K, Nogawa M, Yuasa T, Egawa $H$, Tanaka $K$, Maekawa T: Rapid quantitation of immunoglobulin $\mathrm{G}$ antibodies specific for blood group antigens A and $\mathrm{B}$ by surface plasmon resonance. Transfusion 2005;45:56-62.

16 Nguyen XD, Klüter H: The SASPA (simultaneous analysis of specific platelet antibodies) assay: implementation and performance in routine laboratory use. Transfus Med Hemother 2006;33:254-259. 